RU-97-86, SU-ITP-97-26, UTTG - 25 -97, PUPT-1742

\title{
Schwarzchild Black Holes in Matrix Theory II
}

\author{
T. Banks ${ }^{1}$, W. Fischler ${ }^{2}$, I.R. Klebanov ${ }^{3}$, L. Susskind ${ }^{4}$ \\ ${ }^{1}$ Department of Physics and Astronomy \\ Rutgers University, Piscataway, NJ 08855-0849 \\ banks@physics.rutgers.edu \\ ${ }^{2}$ Theory Group, Department of Physics \\ University of Texas, Austin, TX, 78712 \\ fischler@physics.utexas.edu \\ ${ }^{3}$ Joseph Henry Laboratories \\ Princeton University, Princeton, NJ 08544 \\ klebanov@puhep1.princeton.edu \\ ${ }^{4}$ Department of Physics \\ Stanford University, Stanford, CA 94305-4060 \\ susskind@dormouse.stanford.edu
}

We present a crude Matrix Theory model for Schwarzchild black holes in uncompactified dimension greater than 5. The model accounts for the size, entropy, and long range static interactions of black holes. The key feature of the model is a Boltzmann gas of D0 branes, a concept which depends on certain qualitative features of Matrix Theory which have not previously been utilized in studies of black holes.

October 1997 


\section{Introduction}

In this paper we will present a crude and simple quantum mechanical model of Schwarzchild black holes in noncompact spacetimes of dimension $11 \geq D \geq 6$. The model accounts for many of the gross properties of a black hole: its mass, entropy, radius, as well as the Newtonian gravitational interaction between two black holes. We hope that future investigations will provide more evidence that it indeed describes black holes.

The model is based on the recent Matrix Theory [1] proposal for a nonperturbative, light cone frame formulation of $\mathrm{M}$ theory. This proposal is far from complete. In particular, it has proven extraordinarily difficult to find a general description of Kaluza-Klein compactification of Matrix Theory. We will try to formulate our model in a way which uses as few of the detailed properties of Matrix Theory compactification as possible.

The essence of our black hole model is simplicity itself: for the most part, the black hole consists of a Boltzmann gas of D0 branes 娄 1

interacting via the long range (IMF)-static forces which are associated with graviton exchange with zero longitudinal momentum. The phrase "for the most part" means that the bulk (at least a finite fraction) of the entropy and energy of the black hole is accounted for by the D0 brane gas. Furthermore, the Schwarzchild radius of the hole is determined by the quantum wave functions of the D0 branes. The other constituents of the hole lie within its Schwarzchild radius. We will also show that the long range static Newtonian interaction of two equal mass black holes (the technical reason for considering only equal masses will be explained below) can be completely understood in terms of the velocity dependent forces between D0 branes and their velocity distribution inside the black hole.

We have mentioned other constituents of the black hole. The necessity for such follows from our insistence on a Boltzmann gas of zero branes. D0 branes are bosons (and fermions). Their statistics symmetry is the residuum of a continuous gauge symmetry which is restored when they are very close together. We will see that a Bose or Fermi gas does not have enough entropy to account for the properties of black holes. The Boltzmann nature of the D0 branes inside a black hole derives from the existence of a classical background configuration of the matrix model. The requisite D0 branes are a certain class of matrix fluctuations around this background. The background completely breaks the gauge invariance of the model, including the would be statistics symmetry of the zero branes. In

1 After submitting this paper, we were informed by I. Volovich of a previous paper in which black holes are treated as a Boltzmann gas of D0 branes, [3] 
a picturesque language which we will explain below, the zero branes are tethered to the background in a way which makes them distinguishable.

The background must satisfy a number of properties in order for the model to make

sense. It is in trying to verify the existence of appropriate backgrounds that we must appeal to our rather imperfect knowledge of the detailed dynamics of the compactified matrix theory. We have been able to make progress on this problem only when $D=11$, or 8 and we have some partial results for $D=7$. Furthermore, beginning with $D=5$ there seems to be a serious problem with our ideas, and when $D=4$ the logarithmic nature of the transverse interactions between D0 branes presents us with some unresolved questions. In order to clearly separate these issues from the central simplicity of our model, we have decided to present things in the following order. In the next section, after recalling some facts derived in a previous paper, [4], we use the Boltzmann gas model to derive some of the properties of black holes. We then present the general criteria for backgrounds which could justify the Boltzmann gas model, and present examples of such backgrounds in 11 and 8 dimensions. Finally, we explain the difficulties in $D=4,5$. Our approach throughout is based on a rule of thumb motivated in [4] which suggests that for the study of Schwarzchild black holes, the optimal value of $N$ (the value which is large enough to obtain an adequate description without involving many redundant variables) is of order the entropy, $S$, of the black hole. In the appendix we discuss some highly speculative arguments which suggest that the appropriate Matrix Theory setting for the study of black holes in $D=4,5$ and perhaps even 6 is the regime where $N \gg S$.

\section{The Boltzmann Gas Model}

We begin by recalling some results of [4] . Our basic strategy is to work in the light cone frame with a compactified lightlike direction of circumference $R$ and total longitudinal momentum $N / R$. As we increase $N$, the number of degrees of freedom in the model increases. The optimal choice of $N$ for describing any given system depends on the system. If we choose $N$ too small we do not describe the system adequately, while if we choose it too large we include many redundant variables which are frozen into their ground state in the system under consideration. Our criterion for fixing the optimal value of $N$ for a system described by a classical spacetime geometry is that the geometrical size of the system, $R_{S}$, must, after a longitudinal boost to momentum $N / R$, fit into the compactification radius. This gives the equation

$$
\frac{M R}{N} R_{S}=R
$$


Or

$$
N=M R_{S}
$$

where $M$ is the mass of the system. This is the minimal value of $N$ which can give us an adequate description of the system, and larger values of $N$ give us a description with many redundant variables. For a generic system there is an as yet unresolved ambiguity about which geometrical parameter $R_{S}$ to use in this equation, but for Schwarzchild black holes $R_{S}$ is clearly the Schwarzchild radius, since there is no other length scale in the Schwarzchild geometry. In this case it is easy to see that $N$ is the entropy of the black hole (all such statements are meant as order of magnitude estimates, no precise numerical coefficients are computed in this paper).

The other result of [4] which we wish to recall is the description of Hawking particles in the light cone frame. In the rest frame, a typical Hawking particle has energy $T_{H} \sim M^{-\frac{1}{D-3}}$ and isotropically distributed momenta of the same order of magnitude. After the boost it has longitudinal momentum $\frac{N}{M R} T_{H} \sim 1$. Its transverse momentum is of order $T_{H}$ and its light cone energy is of order $\frac{M R}{N} T_{H} \sim N^{-\frac{2}{D-2}}$. We will see that these are precisely the kinematic properties of one of our Boltzmann D0 branes.

We are now ready to present our model. For concreteness, we restrict attention to toroidally compactified matrix models. We believe that our considerations will turn out to be more general than that, since they rely only on very general properties of the model. In particular, in all known versions of Matrix Theory, momentum in the noncompact directions is carried only by $D N$ degrees of freedom $X_{a}^{i}$ which represent the positions of $N$ D0 branes in the noncompact dimensions. Due to the gauge invariance of the underlying model, there is some ambiguity about how these D0 brane positions are extracted from the full set of degrees of freedom. The role of the background, which we discuss in the next section, is to resolve this ambiguity. For the moment, we simply assume that the $X_{a}^{i}$ can be thought of as the coordinates of distinguishable particles.

When the D0 branes are far apart, one can calculate an effective interaction between them [5] which has the form (if the D0 brane velocities are slow, which we will verify self consistently in a moment):

$$
H=\sum_{a=1}^{N} \mathbf{p}_{a}^{2}+A G_{N} \sum_{a, b} \frac{\left(\mathbf{p}_{a}-\mathbf{p}_{b}\right)^{4}}{\left|\mathbf{X}_{a}-\mathbf{X}_{b}\right|^{D-4}}
$$

When $D=4$ the power law interaction is replaced by a logarithm. $G_{N}$ is the dimensionally reduced Newton constant, and the coefficient $A$ was calculated in [5]. This is indeed 
the correct interaction between uncompactified gravitons in $D$ spacetime dimensions. In Matrix Theory it is calculated by integrating out the Super Yang Mills degrees of freedom other than the D0 brane coordinates.

Now consider a metastable configuration in which all $N$ D0 branes are bound together. In the spirit of the mean field approximation, each $D 0$ brane should have approximately the same mean distance $R_{S}$ from the center of mass, and (by the uncertainty principle) the same mean momentum $1 / R_{S}$. Equating the kinetic and potential terms in the Hamiltonian of the bound system (the virial theorem), we obtain

$$
B G_{N}^{-1} R_{S}^{D-2}=N
$$

The numerical constant $B$ cannot be calculated with our present crude methods. We are treating the system of D0 branes as a Boltzmann gas. Thus its entropy is of order $N$. We have thus derived the Bekenstein-Hawking area law (up to a numerical constant) from the basic principles of Matrix Theory.2.

We can also calculate the energy of the system to the same accuracy. The single particle energies are of order $R_{S}^{-2}$, so the total energy scales like $N^{1-2 /(D-2)}$. Using the standard relation between light cone energy and mass, we find that the mass of the system scales like

$$
M \sim G_{N}^{-1 /(D-2)} N^{1-1 /(D-2)}=G_{N}^{-1 /(D-2)} N^{(D-3) /(D-2)}
$$

Again, the dependence on Newton's constant follows from dimensional analysis and the fact that the radius, $R$, of the lightlike circle is only an overall scale in the Hamiltonian. Thus, the correct relation between mass and Schwarzchild radius also follows from our model.

Note that the kinematics of the individual D0 branes (transverse momentum of order $R_{S}^{-1}$, longitudinal momentum of order one), is precisely that of boosted Hawking particles. Thus it is tempting to identify the elementary process of Hawking radiation as the emission of a single D0 brane from the bound cluster. In order to discuss the rate of this process,

2 There is a subtle loophole in this argument which we will return to when we discuss backgrounds in the next section .

3 As this paper was in preparation, we received preprints by Horowitz and Martinec [6] and by $\mathrm{Li}$ [7], which also derive the area law in arbitrary dimensions. These authors also claim to calculate the entropy as order $N$, but do not make explicit the fact that one must treat the D0 branes as distinguishable. 
we will have to say something about the nature of the backgrounds. Another consequence of these kinematical relations is the necessity for treating the D0 branes as a Boltzmann gas. The energy per particle, or effective temperature, is of order $R_{S}^{-1}$ and goes to zero with $N$. In a low temperature Bose or Fermi gas, the entropy per particle vanishes as a power of the temperature. Thus, we must assume Boltzmann statistics for the zero branes in order to reproduce the entropy of black holes.

As a final application of our model, we will compute the Newtonian gravitational potential between a pair of equal mass static black holes 4 . The restriction to equal mass is a consequence of our strategy of choosing an optimal value of $N$ to study any system. Black holes of different masses have very different numbers of excited degrees of freedom. We cannot give an adequate description of the combined system without "overdescribing" the black hole of smaller mass. We have yet not understood how to study such a system in the matrix model.

Another important qualification is that present Matrix Theory technology restricts us to the study of processes with zero longitudinal momentum transfer. Thus rather than studying black holes at fixed positions in their common rest frame, we must average the potential over the longitudinal circle. This introduces an interesting subtlety into the calculation: the length of the averaging circle in the boosted frame is Lorentz contracted relative to that in the rest frame. We will do a calculation of the light cone energy of the two black hole system in the boosted frame. We then use the usual connection between light cone energy and rest mass to calculate the rest energy, and thus the static potential of the system. This must be interpreted as the Newtonian potential averaged over a longitudinal circle whose size is related to that in the boosted frame by a Lorentz contraction factor.

To compute the light cone energy of a pair of black holes, we take into account only the velocity dependent interactions between their zero brane constituents:

$$
\delta P^{-}=A G_{N} \sum_{a, b} \frac{\left(\mathbf{p}_{\mathbf{1}_{a}}-\mathbf{p}_{\mathbf{2}_{b}}\right)^{4}}{\left|\mathbf{X}_{\mathbf{1}_{a}}-\mathbf{X}_{\mathbf{2} b}\right|^{D-4}}
$$

This should be averaged over the velocity distribution of the two holes. Many terms drop because of spherical symmetry of the distributions and we are left with

$$
\delta P^{-} \sim N^{2} \frac{G_{N}}{r^{D-4}}\left(G_{N} N\right)^{\frac{-4}{(D-2)}}
$$

4 The idea for this calculation is due to S.H.Shenker, who also collaborated in the actual computations. 
where we have used $r$ to denote the distance between the centers of mass of the two black holes and inserted our result for the average velocity. This is to be compared with

$$
\delta P^{-}=\frac{(2 M+V(r))^{2}}{N}-4 \frac{M^{2}}{N}=8 \frac{M}{N} V(r)
$$

We obtain

$$
V(r) \sim G_{N} \frac{N^{3}}{M r^{(D-4)}}\left(G_{N} N\right)^{\frac{-4}{(D-2)}}
$$

Using the Bekenstein-Hawking mass-entropy relation (which we have derived above), we get:

$$
V(r) \sim G_{N} \frac{M^{2}}{r^{(D-4)}}\left(G_{N} N\right)^{-\frac{1}{(D-2)}}
$$

This is the correct formula for the static Newtonian potential appropriately averaged over the longitudinal circles. To see this note that the last factor is $1 / R_{S}$. Indeed, the averaging should introduce a factor of $1 / R$, and if we are in the rest frame of the black holes and work with $S \sim N$, then $R \sim R_{S}$.

An important question is whether the more complicated multibody forces which occur at higher orders in the matrix model perturbation expansion can effect the calculations which we have done. For example, the three body interaction computed by Dine and Rajaraman 8$]$ scales like $N^{3} \frac{v^{6}}{r^{14}}$ in eleven dimensions. Note that for values of $v \sim \frac{1}{r} \sim N^{-(1 / 9)}$ this has the same order of magnitude as the terms we have included in our analysis. although a quantitative analysis of the black hole will have to take into account all sorts of complicated multibody interactions, the scaling laws which we have derived will still be obeyed.

\section{Background Information}

We now turn to the central question of why it is proper to treat D0 branes as Boltzmann particles. The basic intuition is that, in the matrix model, particle statistics is part of a larger gauge group, which acts on degrees of freedom in the theory which are not particle like. They do not carry momentum in the spacetime of the matrix model, but may be viewed as internal excitations of the partons which do carry such momentum. In

5 This observation is due to S.H.Shenker, who informs us that the general term in the perturbation expansion obeys this scaling rule. It is of the form $N^{k} \frac{v^{2 k}}{r^{7(k-1)}}$. 
some sense, this is one of the key feature which distinguishes Matrix Theory from quantum field theory (the other is its holographic nature).

Consider a background configuration , $M_{c l}$ of Matrix Theory described by some fixed value of the matrix variables, $M_{c l}$. In particular, it will have fixed values $\mathbf{X}_{c l}$ of the position variables whose zero mode is shifted by a multiple of the unit matrix under spatial translations. The background must satisfy a number of properties:

1. It must be a metastable, classical configuration of the matrix model, i.e. the center of a coherent state with long lifetime.

2. It must not be left invariant by any element of the gauge group. As a consequence of the first postulate, this leads to a situation in which the gauge group is "spontaneously broken". Since we are always dealing with a finite quantum system, this means that the time scales for motions of the collective coordinates which gauge rotate the classical background are much longer than any of the other time scales in the system.

We then define a configuration of "zero branes in the classical background $M_{c l}$ " as the configuration in which we shift the position coordinates by

$$
\mathbf{X}_{c l} \rightarrow \mathbf{X}_{c l}+\sum_{a=1}^{N} \mathbf{x}^{\mathbf{a}} \delta_{a}
$$

where $\delta_{a}$ is a collection of $N$ commuting $N \times N$ matrices which we will specify more precisely in a moment.

All compactifications of Matrix Theory whose Hamiltonian is understood contain a term

$$
-\operatorname{Tr} \operatorname{tr}\left[X^{i}, X^{j}\right]^{2}
$$

where the $X^{i}$ are related to coordinates in the noncompact space dimensions. They are $N \times N$ matrices whose matrix elements are operators in another Hilbert space. The small $t r$ refers to the trace in this internal space, while $\operatorname{Tr}$ is the matrix trace. Such a term will give rise to harmonic potentials for the coordinates $\mathbf{x}^{\mathbf{a}}$ in the classical background $\mathbf{X}_{\mathbf{c l}}$. For a given classical background, we will choose the $\delta^{a}$ in such a way as to minimize the coefficients of these harmonic potentials. We will give examples of this below in particular dimensions, and argue that it is always possible to make these terms as small as $\frac{1}{N} \sum \mathbf{x}^{\mathbf{a}^{2}}$. If the dimension $D>5$ then it is easy to see that, for distances of order $R_{S} \sim\left(G_{N} N\right)^{1 /(D-2)}$, 
the harmonic energy is no larger than the energies associated with zero brane interactions 0 (in $D=6$ the two energy scales are the same).

We will impose two more constraints on the classical background:

3. The total energy of the background configuration should be smaller than or equal to the total zero brane energy $N^{1-\frac{2}{D-2}}$ in dimensionally reduced Planck units.

4. The classical size of the background, should be smaller than or equal to the Schwarzchild radius. We will comment on this condition further below.

If we can find backgrounds which satisfy all of these conditions then we can remedy the difficulties of the zero brane model of black holes which we presented in the previous section. That model actually had two inconsistent features. The first of these was the treatment of the zero branes as if they were distinguishable particles. This is resolved by the existence of the background. Mathematically, the configurations described by Equation (3.1) have no residual $S_{N}$ gauge symmetry which could act as particle statistics. Intuitively, the interactions with the background distinguish the zero branes from each other. We will see a particularly vivid physical picture of this in the eleven and eight dimensional examples.

The second unclear feature of the model of the previous section was the assumption of a bound configuration of zero branes. The matrix Hamiltonian has only one exact bound state configuration - the supergraviton with longitudinal momentum $N$. This is a threshold bound state. Every other state in the theory eventually breaks up into separated asymptotic supergraviton states (the asymptotic supergravitons with longitudinal momenta smaller than $N$ are also stable excitations of the model, but they do not correspond to a bound configuration of $N$ zero branes). The background makes explicit the necessity of finding metastable excitations of the system. Once the metastable background configuration is present, its zero brane excitations are bound to it by harmonic forces. It is only via the quantum processes which allow the background to fluctuate that the system can decay.

The model of metastable classical background plus zero branes predicts the correct relation between the mass and Schwarzchild radius of a black hole, as well as the gravitational interaction between black holes of equal mass. We would like to claim that it

6 Note that in principle we should rederive the two body zero brane interactions in the presence of the background, by calculating the one loop quantum fluctuations around the configuration (3.1) . Since the background is supposed to receive only small quantum corrections itself, it seems obvious that for large $\mathbf{x}^{\mathbf{a}}$ the result will be the same as that in the absence of the background, but this point should be checked. 
also predicts the correct Bekenstein-Hawking mass entropy relation for black holes. In order to do this we must understand the entropy coming from summing over background configurations. It should turn out to be less than or equal to the zero brane entropy. We do not have a general or rigorous understanding of why this is so. A complete answer to this question would constitute a proof of the Bekenstein bound on entropy in the context of the matrix model (in [1] it was argued only that the bound was satisfied by the ground state wave function). We do not have such a proof. In dimensions 11 and 8 we believe that we understand the entropy of background configurations satisfying the conditions outlined above. This shows that the configurations which we study do satisfy the entropy relation for black holes. We do not have an argument that configurations other than the ones we have studied cannot pack larger entropy into the same area.

Another issue is raised by a discussion of the entropy of backgrounds. Any configuration satisfying the criteria we have outlined will behave like a black hole. But what is a typical background? In particular, if there are background configurations with entropy of order that of the zero branes, then they must be understood if we ever wish to compute the coefficient in the Bekenstein-Hawking formula. We cannot make any more progress on these questions without turning to particular examples.

\section{Schwarzchild Black Holes in Eleven Dimensions}

In eleven noncompact dimensions, it is natural to search for the semiclassical backgrounds of our black hole model among the membrane excitations of the eleven dimensional matrix model. The latter are semiclassical excitations of the model whose quantum metastability is guaranteed by their closeness to exact BPS configurations [1] [9]. Let us remind the reader of how these configurations are constructed. The matrices

$$
U^{k} V^{l}
$$

where $U$ and $V$ are unitary matrices satisfying $U^{N}=1=V^{N}$, and

$$
U V=e^{\frac{2 \pi i}{N}} V U
$$

are a complete set of $N \times N$ matrices. For large $N$ we concentrate on those matrices whose expansion

$$
M=\sum M_{k l} U^{k} V^{l}
$$


approaches the Fourier expansion of a smooth function on a torus with coordinates $p$ and $q$ in the interval $[0,2 \pi]$. The identification is made via the formal substitution $U \rightarrow$ $e^{i q}, V \rightarrow e^{i p}$. For such matrices commutators approach $\frac{2 \pi i}{N}$ times the Poisson bracket of the corresponding functions, and the trace approaches $N$ times the integral over the torus. The matrix model action transmutes under these formal substitutions into the action of the light cone supermembrane [10].

A membrane configuration is simply a choice of the $\mathbf{X}$ coordinates of the matrix model to be such smooth functions on the torust A generic such choice will break all of the $U(N)$ gauge symmetry. Furthermore, it is clear from the construction that for a large smooth membrane these are long lived semiclassical states.

Now consider the matrices defined by the following periodic Gaussian functions

$$
\Theta_{p_{i}, q_{i}}=\sum_{k, l} e^{-N\left[\left(p-p_{i}-2 \pi k\right)^{2}+\left(q-q_{i}-2 \pi l\right)^{2}\right]} .
$$

For large $N$, the commutator of two of these matrices is well approximated by the Poisson bracket and is smaller than

$$
e^{-\frac{1}{2} N\left[\left(p_{i}-p_{j}\right)^{2}+\left(q_{i}-q_{j}\right)^{2}\right]}
$$

Taking a distribution of points $\left(p_{i}, q_{i}\right)$ spaced by distances of order $1 / \sqrt{N}$ we can obviously make this commutator as small as we like. There are $o(N)$ such points and so we can find $o(N)$ matrices $\delta_{a}$ of this form.

The $\delta_{a}$ are normalized to $\operatorname{Tr} \delta_{a}=1$ They are the "cells in phase space" which make up individual D0 branes. Note that we have implicitly used the classical membrane configuration to define the $\delta_{a}$. We cannot do unitary transformations on $\delta_{a}$ which leave the membrane configuration unchanged. Thus, there is no statistics symmetry as there is for D0 branes in free space. Each zero brane is "tethered" to some particular point in the membrane volume.

We can now compute the harmonic potential between the zero branes and the membrane by plugging the configuration $X_{c l}+\sum x^{a} \delta_{a}$ into the membrane Hamiltonian. The

7 We choose the torus only for simplicity of exposition. Actually for sufficiently large $N$ we can find matrices reproducing membranes of any finite genus with any stated accuracy, and in principle any such background will serve the same purpose. For fixed $N$, only a finite number of genera will be well approximated. 
terms bilinear in different $\delta a$ are of the same order as the commutator between different D0 branes, and so we drop them. The total harmonic potential is thus

$$
\delta H \sim \sum\left(\mathbf{x}^{\mathbf{a}}\right)^{2} N \int d p d q\left[p^{2}+q^{2}\right]\left(\nabla X_{c l}\right)^{2} e^{-N\left(p^{2}+q^{2}\right)} .
$$

The factor $N$ in front of the integral comes from the $1 / N$ in the definition of the energy in terms of membrane variables, and two factors of $N$ originating in the derivatives of the $\delta_{a}$. For truly smooth membranes, the gradient of $X_{c l}$ is independent of $N$ as $N \rightarrow \infty$. The harmonic potential then has an overall coefficient $1 / N$. It will also be useful to consider configurations $X_{c l}$ whose gradients scale like some positive power of $N$. In principle, if we wanted to use these in the construction of black holes, we would have to investigate their stability properties, since it is no longer clear that they are close to being BPS states. In the end, we will discard such configurations for other reasons so we will not stop to perform this investigation. The harmonic potential for such a configuration is larger by a factor of $k^{2}$, where $k$ is its maximum wavenumber, than that for a smooth membrane. In any event, we must have $k<\sqrt{N}$. For larger values of $k$ the membrane description of matrices is completely wrong. There are no matrices corresponding to such short wavelength membrane configurations.

We have now essentially completed the construction of a black hole model in eleven dimensions. The energy of smooth membrane configurations is of order $1 / N$ and thus negligible compared to the energy $N^{7 / 9}$ (or even to the energy per particle, $N^{-2 / 9}$ ). The harmonic potential experienced by a single zero brane at the Schwarzchild radius $R_{S} \sim$ $N^{1 / 9}$ is of order $N^{-(7 / 9)}$, also much less than the energy per particle of the gas of interacting zero branes. Finally, the entropy of all possible smooth membrane configurations can be estimated as that of a cutoff $2+1$ dimensional quantum field theory, with cutoff independent of $N$. The entropy is thus of order 18 and makes a negligible perturbation to the zero brane entropy $N$.

We can try to find a larger set of configurations by allowing $k$ to grow with $N$. The condition that the harmonic potential not interfere with the interacting zero brane dynamics is $k^{2}<N^{5 / 9}$. The membrane entropy is of order $k^{2}$ (volume of the field theory in cutoff units), and is still negligible compared to the zero brane entropy. In order to have entropy of order $N$ from membrane configurations, we would have to consider membranes

8 For each membrane we can sum over all configurations of the D0 branes, so the membrane and zero brane entropies should be added. 
with a wave number cutoff of order $\sqrt{N}$. This is the edge of validity of the membrane

picture 9. These configurations have energy at least as large as $N$ and do not satisfy the black hole mass-entropy relation. Their mass is at least $N$, larger by a factor $N^{1 / 9}$ than that of a black hole of similar entropy. The Schwarzchild radius of a black hole of this size will not fit into the compactification volume. It is not easy to obtain a first principles matrix model estimate of the size of these configurations. A normal $2+1$ dimensional field theory estimate of the mean square fluctuation of the membrane coordinate gives $<x^{2}>^{\frac{1}{2}} \sim N^{1 / 4}$. This is much larger than the Schwarzchild radius of a black hole of mass $N$ in 11 dimensions. We suspect that, if anything, this is an underestimate of the fluctuation of the membrane coordinate in this cutoff, nonrenormalizable field theory.

Thus, among membrane configurations of the matrix model, none can be found which compete with the membrane plus zero brane gas in putting a large amount of entropy into a small area. If we could demonstrate the same thing for all other states of the matrix model, we would have proven the Bekenstein bound in eleven dimensions.

\section{Eight Dimensional Black Holes in Matrix Theory}

We have studied the system with eight noncompact dimensions in [4] Here we elaborate the discussion and take a somewhat different point of view. Our general approach in the current paper suggests that we look for a classical background to distinguish the zero branes. We can of course study the same type of background which we used in eleven dimensions - membranes. However, we will find a much more common type of background in the eight dimensional version of the theory, one which contributes a finite fraction of the energy and entropy of the black hole. Indeed, the typical background is described by the configurations we studied in [4] .

In order to have entropy of order $N$ and carry a finite fraction of the energy, the background must consist of $o(N)$ independent excited degrees of freedom, each carrying an energy of order the temperature, $N^{-(1 / 3)}$. This means that most of the degrees of freedom of the $3+1$ dimensional SYM theory are irrelevant for these considerations, for they carry energy of order one. The relevant DOF are those of the uncompactified model, except that the coordinates in the compactified directions are angle variables, since they

9 It should be clear that such configurations could not be taken as backgrounds in our model. We are discussing them merely as an example of high entropy configurations which are not contained within the Schwarzchild radius. 
originate as Wilson loops of the $3+1$ gauge fields. The first component of the background is a classical expectation value of these Wilson loops corresponding to a more or less uniform lattice of D0 brane positions on the three torus, with spacing $N^{-(1 / 3)}$. This background breaks the gauge symmetry down to $U(1)^{N}$, the subgroup preserving the basis in which the Wilson loop is diagonal. Note in particular that there is no permutation gauge symmetry among the eigenvalues. Each eigenvalue is labelled uniquely by its position on the three torus. The energy of this configuration of Wilson loops is of course zero. We will call off diagonal matrix elements in the basis in which the Wilson loop is diagonal, charged fields. Those corresponding to gauge field components will be called $\mathrm{W}$ bosons, and those corresponding to noncompact position coordinates will be called charged Higgs fields.

In the presence of the Wilson loop, the charged W boson and Higgs fields (the components of off diagonal matrices in and perpendicular to the three torus respectively) have no zero energy modes. The $I J$ matrix element 10 feels a potential $A_{I}-A_{J}$. For points separated by $o(1)$ links of the lattice, this is of order $N^{-(1 / 3)}$. Of course this is also the energy of the lowest lying mode of this variable. Thus, ignoring for the moment the term in the Hamiltonian quadratic in commutators of the charged fields with each other, we should be able to excite each of these degrees of freedom to a classical state with energy of order the temperature. Thus, for each D0 brane we excite $o(1)$ of these degrees of freedom (its links to a few nearest neighbors). This gives the background an entropy of order $N$ there are $e^{c N}$ different backgrounds with the same characteristics.

The zerobrane positions in the noncompact directions are the diagonal matrix elements $r^{a}$ (with $1 \leq a \leq 6$ ) of the Higgs fields. Note that because of the nature of the Wilson loop there is no residual $S_{N}$ gauge symmetry permuting these variables. The harmonic potential for these variables has the form

$$
<\left|W_{I J}\right|^{2}>\left(r_{I}^{a}-r_{J}^{a}\right)^{2}
$$

where the brackets denote averageing over the ensemble of backgrounds. There are actually two terms of this form, one coming from the charged Higgs field background and the other from charged W bosons, but they have the same general nature, so we lump them together. We will take the individual matrix elements $W_{I J}$ to be of order one. To understand the order of magnitude of the harmonic potentials, begin with the special configuration where

10 We use multiindex notation. Capital letters refer to triplets of integers specifying positions on the zerobrane lattice. 
the matrix elements are equal to one only between nearest neighbor points on the toroidal lattice of zero branes and zero elsewhere. Then the frequency squared matrix for the set of coupled oscillators looks like the Laplacian on a three dimensional toroidal lattice of side $N^{(1 / 3)}$. The nonzero eigenvalues of this matrix are thus of order $N^{-(2 / 3)}$. The eigenvectors represent displacements of the zero branes away from the center of mass of the system. When these displacements are of order the Schwarzchild radius, that is $o\left(N^{1 / 6}\right)$, these harmonic energies are of the same order as the single particle energies of the zero branes computed in the mean field approximation with their velocity dependent two body interactions. Thus, a consistent picture emerges, in which both harmonic attraction to the background and interparticle forces bind the zero branes at a distance of order the Schwarzchild radius from the center of mass.

Small changes of individual $W_{I J}$ matrix elements are equivalent to changing the Laplacian on a flat toroidal lattice to that on a curved background. The inclusion of nonzero matrix elements between nonnearest neighbors (but always a distance of order one away from each other) adds higher derivative terms to the Laplacian and does not change the qualitative scaling behavior for large $N$.

Finally, we must check the terms in the energy coming from the square of the commutator of charged fields. Consider for example matrices whose only nonzero matrix elements are between nearest neighbors on the toroidal lattice. If all of the nearest neighbor matrix elements were equal, the matrix would be a sum of commuting shift operators on the torus. Thus, the commutator of two such local matrices is proportional to differences of nearest neighbor matrix elements. The Hamiltonian is proportional to the trace of the square of the commutator. If we take nearest neighbor matrix elements of order one, with differences between them of order $N^{-(1 / 6)}$, then the energy will be a sum of $N$ terms, each of order $N^{-(1 / 3)}$. Clearly, these estimates generalize to matrices with nonzero matrix elements for $o(1)$ next to nearest neighbors. Thus, it is possible to find classical configurations of charged fields whose energy and entropy are of the same order as those of the zero branes.

It is interesting that the background entropy is so low in eleven dimensions but appears to be a finite fraction of the total black hole entropy in eight. We do not have a clear picture of the significance of this result, and we do not know how it generalizes to other dimensions. In seven dimensions, although the compactified theory is poorly understood, we believe that we have identified background configurations involving tensionless strings, which have energy per zero brane of order $N^{-(2 / 5)}$. This suggests that high entropy backgrounds will be found in all dimensions below nine. However, we do not know how to compute the 
harmonic restoring force in this case and the analysis is too preliminary to be presented here.

If the background contributes a finite fraction of both the energy and entropy of the black hole, it is somewhat artificial to separate the system into zero branes plus background. We suspect that this may be the general case when the noncompact dimension is less than nine. Nonetheless, we have chosen to present the statistical mechanics of matrix black holes in terms of the zero brane gas, because this makes it clear that there will be configurations satisfying the black hole mass-entropy-radius relation for Matrix Theory compactifications to any dimension greater than five. Even in eight dimensions, we can find backgrounds with harmonic potentials of order $1 / N$ and build black holes from them. They are simply much less numerous than the configurations studied in [4] .

It is in four and five uncompactified dimensions that our approach really appears to run into trouble. We have so far been unable to find configurations which produce a harmonic potential with coefficient smaller than $1 / N$. In $D$ dimensions, at the Schwarzchild radius, this gives an energy of order $N^{\frac{2}{D-2}-1}$, which should be compared to the energy per particle of the D0 brane gas $N^{-\frac{2}{D-2}}$. The ratio of energies is $N^{\frac{6-D}{D-2}}$. Thus, in four and five dimensions the harmonic potential dominates and our picture breaks down.

Actually, in four dimensions, this discussion is altogether too naive. Indeed it is completely unclear whether the entire matrix model formalism makes sense in four dimensions. In the matrix model we are supposed to treat four dimensions by first compactifying to three and then decompactifying the longitudinal direction by taking $N$ to infinity. But in three dimensions, Kaluza Klein modes have long range dilaton fields which render their BPS charges undefined. The energy of a generic collection of BPS Kaluza-Klein modes appears to be infinite. We suspect that this is a deep property of $M$ theory which implies that compactifications below four dimensions only make sense in a cosmological context. A discrete light cone approach could only make sense above four dimensions. This is clearly not the time or place to discuss such issues.

This leaves us with five dimensional compactifications of the matrix model. We are unsure whether the difficulty which our model encounters indicates the necessity of finding special background configurations with small harmonic potentials for zero branes, or a deeper sickness in the five dimensional theory. In the Appendix we suggest that the sickness may be avoided in the regime $N \gg S$. 


\section{Conclusions}

We have been led to a remarkably simple picture of Schwarzchild black holes in matrix theory, which appears to be valid when there are more than five noncompact dimensions. They are collections of zero branes, interacting with themselves and with a classical background. In eleven dimensions the background appears to be necessary only to "Boltzmannize" the zero branes, that is to break their statistical gauge symmetry. It does not contribute appreciably to the energy or entropy of the black hole. In eight dimensions, the background does make substantial contributions to the entropy, and we expect that this may be the general case with eight or fewer compact dimensions.

One obvious calculation which we have not done is the Hawking evaporation rate. Given the parallel between the kinematics of Hawking particles and our D0 branes, a natural guess for the Hawking evaporation mechanism is a quantum fluctuation which erases the part of the classical background which interacts with a given D0 brane. In our 8 dimensional model for example, this consisted of $o(1)$ strings. We have not yet been able to estimate the probability for these strings to disappear and we are not able to understand why it should decrease like a power of $N$ (let alone compute the power). If indeed this probability falls like an appropriate power of $N$ then we could understand the Hawking evaporation formula from the microscopic mechanics of our model. This is an interesting topic for further study.

Apart from the obvious call to solve the mysteries of low dimensional compactification,

our approach should be extended in various directions. One should obtain a clear picture of the relevant background configurations for all $D>5$. One should also extend our considerations to charged and rotating black holes. Perhaps most interesting of all would be to model the experience of an "observer" falling into our matrix black hole, and to extract the spacetime metric which he feels.

Finally, we remark that recent work on scattering in Matrix Theory [8] [11] has made it clear that the correct gravitational physics cannot be extracted from the matrix model at small values of $N$, unless it is protected by SUSY. Although our analysis depended mostly on large $N$ scaling laws, we do not know the extent to which our considerations will be affected by these results. In particular, our estimate of the value of $N$ necessary to reproduce black hole physics, takes no account of the transverse separation of the black holes. It may be that the correct physics can only be obtained in a regime in which the black hole wave functions overlap substantially. In this case our derivation of the 
Newtonian interaction between black holes will appear accidental. We hope that this is not the case, since the Boltzmann gas model is appealing in its simplicity. The best way to attack these questions is to see what other properties of black holes can be derived from the model.

\section{ACKNOWLEDGEMENTS}

T.B. would like to acknowledge conversations with D.Gross, J.Polchinski and G.Horowitz, and the hospitality of ITP Santa Barbara, where those conversations took place in the summer of 1997. TB and WF would like to thank the Physics Department of Stanford University for its hospitality in July and August of 1997 when this work was begun. W.Fischler would like to thank the Physics Dept of Rutgers University for its hospitality during the time that this work was completed. All of the authors would like to thank S.Shenker for collaborating on portions of this work and for making his usual penetrating observations about all of it. The work of TB was supported in part by the Department of Energy under Grant No. DE - FG02 - 96ER40959 and that of W.F. was supported in part by the Robert A. Welch Foundation and by NSF Grant PHY-9511632. The work of I.R.K was supported in part by the DOE grant DE-FG02-91ER40671, the NSF Presidential Young Investigator Award PHY-9157482, and the James S. McDonnell Foundation grant No. 91-48. L.S. acknowledges the support of the NSF under Grant No. PHY - 9219345.

\section{Appendix: The Well Resolved Black Holes}

In section 5 we showed that our approach runs into trouble for black holes in $D<6$. Note that in another approach [12] the search for black holes in matrix theory compactified to five dimensions has led to the prediction that the system has negative specific heat. This is not unexpected because $D=5$ is, of course, the dimension where the effective theory of the matrix model (at least for toroidal compactifications) seems to contain gravitational degrees of freedom [13]. Let us note, however, that the pathologies with black holes have appeared in the regime $N \sim S$ where there are barely enough degrees of freedom to describe them qualitatively. In this section we suggest that they may be avoided for $N \gg S$ where the black holes are well resolved and we may hope for a quantitative description.

Let us see what form of the light-cone equation of state is necessary to reproduce the mass-entropy relation for Schwarzschild black holes. Using $E=M^{2} R / N$, we arrive at

$$
S \sim G_{N}^{\frac{1}{D-3}}\left(\frac{N E}{R}\right)^{\frac{D-2}{2(D-3)}}
$$


The specific heat implied by this is positive for $D>4$.

$D=4$ is a special case where the specific heat is infinite,

$$
S \sim \frac{N G_{N}}{R} E
$$

This behavior is characteristic of a gas of strings whose tension scales as $1 / N^{2}$. Strings of this kind have been conjectured to exist in M-theory compactified on $T^{7}$ [14], but their relevance to this equation of state is unclear.

For $D=5$ (6.1) gives $S \sim E^{3 / 4}$, which is characteristic of a $3+1$ dimensional massless field theory. In Matrix theory compactified on $T^{6}$ there are 3 transverse directions. In 15] it was suggested that the 0-branes become smeared in these directions, so that an appropriate description is $3+1$ dimensional. Our comparison with Schwarzschild black holes seems to give an independent reason to believe that Matrix theory compactified on $T^{6}$ is described at very low energies by such a field theory.

For $D=6$, (6.1) gives $S \sim E^{2 / 3}$, which is characteristic of a $2+1$ dimensional massless field theory. For $D>6$ there are no cases where we find a field theoretic scaling, $S \sim E^{\frac{p}{p+1}}$.

It is interesting that, only in cases where the specific heat is infinite or negative for $N \sim S$, do we find recognizable scalings for $N \gg S$. Based on this we speculate that the appropriate setting for black holes in $D=4$ and 5 (and perhaps even in $D=6$ ) is to work with $N \gg S$. 


\section{References}

[1] T.Banks, W.Fischler, S.Shenker, L.Susskind, Phys.Rev. D55 (1997) 112, hep-th/9610043.

[2] J.Polchinski, TASI Lectures on D-Branes, hep-th/9611050

[3] I.V.Volovich, Talk given at 2nd International Sakharov Conference on Physics, Moscow, Russia, 20-23 May 1996, hep-th/9608137.

[4] T.Banks, W.Fischler, I.R.Klebanov, L.Susskind, hep-th/9709091.

[5] M.R.Douglas, D.Kabat, P.Pouliot, S.H.Shenker, Nucl.Phys. B485 (1997) 85, hepth/9608024.

[6] G.T.Horowitz, E.J.Martinec, hep-th/9710217.

[7] M.Li, hep-th/9710226.

[8] M.Dine, A.Rajaraman, hep-th/9710174.

[9] T.Banks, N.Seiberg, S.Shenker,Nucl.Phys. B490 (1997) 91, hep-th/9612157.

[10] B. de Wit, J. Hoppe, H. Nicolai, Nucl.Phys. B 305 [FS 23] (1988) 545.

[11] M.R.Douglas, H.Ooguri, S.Shenker, Phys.Lett. B402 (1997) 36, hep-th/9702203; M.R.Douglas, H.Ooguri, hep-th/9710178.

[12] I.R.Klebanov, L.Susskind, hep-th/9709108.

[13] N.Seiberg, hep-th/9710009.

[14] A. Hanany and I.R. Klebanov, Nucl. Phys. B482 (1996) 105.

[15] A. Sen, hep-th/9709220. 